tion of the (vortex-like?) excitations which may have given rise to the much lower critical velocities observed in the flow experiment recently performed by R. M. Mueller, E. B. Flint and E. D. Adams (Phys. Rev. Lett., 36, 1460; 1976) at the University of Florida.

Although a number of important conclusions may be drawn from the present data, the Helsinki experiment -as the authors themselves point outis really only a beginning. Their principal achievement is to have demonstrated the technical feasibility of using ions to probe the superfluid phases of ${ }^{3} \mathrm{He}$. It is to be hoped that future experiments of this type, taken in conjunction with improvements in the theoretical situation, will enable the temperature dependence of the energy gap separating the paired atoms of the superfluid from the (unpaired) excited states of the liquid to be mapped out in detail; it should be possible, using a more carefully designed geometry, to explore the anisotropy of the A phase; and the use of positive ions, to judge from earlier experience in superfluid ${ }^{4} \mathrm{He}$, may perhaps enable completely new types of excitation to be created and identified.

\section{Nitrogen aggregates in diamond}

\section{from John Walker}

As regular readers of News and Views may remember, nitrogen is a common impurity in diamond, though how exactly it got there and the precise forms it adopts within the crystal are only poorly understood. But a recent paper by G. Davies (J. Phys. C : Solid St. Phys., 9, L537-L542; 1976) partly resolves the latter question. The question is important, and not only for scientific reasons since it bears on the synthesis of diamond.

Synthetic diamonds are usually yellow, because they contain a small amount (perhaps one part per million) of dispersed nitrogen. That is, each nitrogen atom is isolated from the other nitrogen atoms, and replaces one carbon atom in the diamond lattice. In the current classification these are type $\mathrm{Ib}$ diamonds. Natural diamonds of this type are rare-one in a thousand.

The majority of natural diamonds, and all the large, well-formed ones suitable for adorning sceptres and film stars' fingers, are what we call type Ia stones. They also contain nitrogen impurity, in quite large proportions (up to almost one per cent); but in contrast to type Ib the nitrogen is aggregated. It appears that in growing her diamonds
Nature did not use the same process as General Electric and De Beers (fortunately for the gem industry, since yellow diamonds, though quite pretty, lack the sparkle and fire of water-white stones).

So what is the form of these aggregates? Until a couple of years ago we thought we knew the answer. The nitrogen gathered in large clusters, many thousands of angstroms in diameter, along $\{100\}$ planes-the "platelets" that Evans and Phaal (Proc. $R$. Soc. Lond., 270, 538-552; 1962) had discovered by electron microscopy. Unfortunately we were wrong. We do not know what the platelets are, but thanks to earlier work by Davies (Nature, 228, 758; 1970) and by E. V. Sobolev et al. (Sov. Phys. Dokl., 12, 665-668; 1967) we know that they contain little if any nitrogen.

We do know that there are at least two different types of aggregate, the so-called A and B forms, which give rise to characteristic infrared and ultraviolet absorption. What Davies has done in his most recent paper is to study one of the A bands, in fact a sharp line, under uniaxial stress. That is, he has squeezed a rectangular diamond block between two anvils, at stresses of the order of 20,000 atmospheres. As Wedlake has remarked, for diamond this is only a romantic squeeze; but it can nonetheless produce interesting results. Although the diamond lattice is cubic, under stress its symmetry is reduced (to tetragonal, trigonal or rhombic depending on the direction of stress), causing absorption bands to split into two or more components (just like the Zeeman effect in atomic spectroscopy-the splitting of spectral lines by a magnetic field).

Davies has deduced from the splitting pattern that the defect responsible for the $\mathrm{A}$ form of nitrogen has trigonal symmetry. The simplest atomic arrangement consistent with this is a pair of nitrogen atoms next to each other ("nearest neighbours"), replacing a pair of carbon atoms in the diamond lattice. Such a model fits in with the other constraints placed by current experimental knowledge: it is not paramagnetic, it is too small to be detected by electron microscopy, and it accounts for the slight increase in lattice parameter observed in nitrogenbearing diamonds compared with pure specimens.

In a further paper (Proc. R. Soc. Lond., A351, 245-265; 1976) Davies and his colleagues, again using uniaxial stress, demonstrate that the well-known H3 absorption and luminescence system in irradiated and annealed diamond has rhombic I symmetry. Davies (J. Phys. C : Solid St. Phys., 5, 2534-2542; 1972) has already shown that the H3 defect is produced when the $\mathbf{A}$ form of nitrogen traps a vacancy or interstitial carbon atom produced during irradiation. Rhombic symmetry follows neatly from the trigonal symmetry of the $A$ aggregate.

What we do not yet know is did the nitrogen aggregate into pairs by diffusion, or was it created like that during synthesis? What is the atomic arrangement of the $B$ aggregate, and is nitrogen involved in the formation of the platelets? We still have many questions left to answer.

\section{Electron transfer systems in microorganisms}

\author{
from D. O. Hall
}

An International Symposium on Electron Transfer Systems in Microorganisms was held at the CNRS Laboratory of Chemical Bacteriology, Marseilles on November 2-5, 1976.

The Marseilles laboratory has been active in the biochemistry of electron transfer in anaerobic bacteria for many years and the meeting was held to discuss the requirements of electron transfer at both the cellular and the molecular level.

The genetics of yeast metabolism has been a fruitful subject for research for some time. P. Slonimski (Laboratory of Molecular Genetics, Paris) discussed some of the recently discovered mitochondrial mutants (MIT ${ }^{-}$) which result in deficiencies in various aspects of mitochondrial metabolism. More than a thousand mutants are now available, opening up the possibility that mitochondrial functions for many different electron transfer reagents and enzymes will be available to biochemists to study the details of electron transport and ATP formation.

B. Haddock (University of Dundee) described his work on electron transfer systems in E. coli and Paracoccus denitrificans. It is becoming clear that

\section{Correction}

In the article "Eukaryotic mRNA: trouble at the 5 -end" (Nature, 263, $188 ; 1976)$ the formula on page 190 was printed incorrectly. The correct formula is given below.

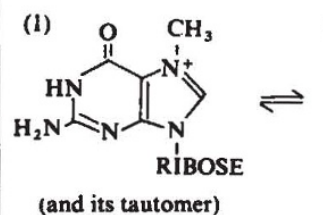<smiles></smiles>

(and its tautomer) 